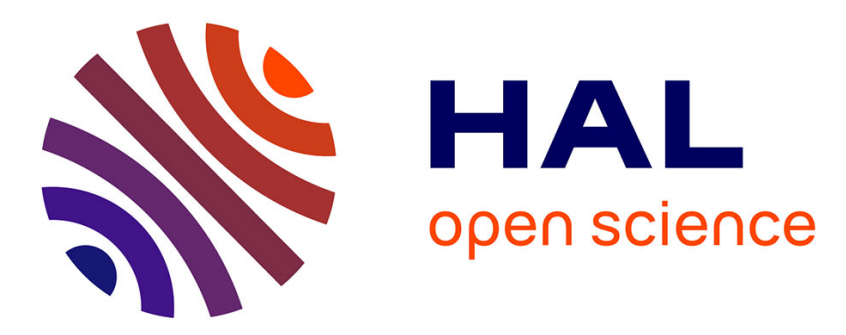

\title{
Generation of a 160-GHz transform-limited pedestal-free pulse train through multiwave mixing compression of a dual-frequency beat signal
}

\author{
Stéphane Pitois, Julien Fatome, Guy Millot
}

\section{- To cite this version:}

Stéphane Pitois, Julien Fatome, Guy Millot. Generation of a 160-GHz transform-limited pedestal-free pulse train through multiwave mixing compression of a dual-frequency beat signal. Optics Letters, 2002, 27 (19), pp.1729-1731. hal-00475084

\section{HAL Id: hal-00475084 \\ https://hal.science/hal-00475084}

Submitted on 21 Apr 2010

HAL is a multi-disciplinary open access archive for the deposit and dissemination of scientific research documents, whether they are published or not. The documents may come from teaching and research institutions in France or abroad, or from public or private research centers.
L'archive ouverte pluridisciplinaire HAL, est destinée au dépôt et à la diffusion de documents scientifiques de niveau recherche, publiés ou non, émanant des établissements d'enseignement et de recherche français ou étrangers, des laboratoires publics ou privés. 


\title{
Generation of 160-GHz transform-limited pedestal-free pulse
}

\section{train through multiwave mixing compression of a dual frequency}

\section{beat signal}

\author{
S. Pitois, J. Fatome and G. Millot \\ Laboratoire de Physique de l'Université de Bourgogne, \\ 9 Avenue A. Savary, B.P. 47 870, 21078 Dijon, France
}

\begin{abstract}
We report the experimental generation of a $160-\mathrm{GHz}$ picosecond pulse train at $1550 \mathrm{~nm}$ using multiple four-wave mixing temporal compression of an initial dual frequency beat signal in the anomalous-dispersion regime of a non-zero dispersion shifted fiber. A complete intensity and phase characterization of the pulse train has been carried out by means of a frequency-resolved optical gating technique, showing that 1.27-ps transform-limited pedestal-free Gaussian pulses have been generated.
\end{abstract}

OCIS codes: $\quad 190.4370,190.4380,320.5520,320.7100$ 
In the context of a rapidly growing traffic in long-haul fiber transmission systems, the development of ultra-short pulse generators enabling high repetition rates and pulse profiles of high quality, will play a crucial role in the performance of time-division-multiplexed communication systems [1]. Since current electronics-based and direct modulation technologies cannot permit the generation of pulses at a repetition rate higher than $40 \mathrm{GHz}$, the nonlinear transformation of a dual-frequency signal in an optical fiber is an attractive method enabling applications in high-speed optical communications. A nonlinear technique based on modulational instability of a pump wave induced by a small signal was first suggested by Hasegawa [2] and realized experimentally by Tai et al. [3]. Another nonlinear method is based on the reshaping of a beat signal which results from the superposition of two pump waves of equal power. Indeed, the nonlinear reshaping of the beat signal into wellseparated short pulses was previously demonstrated by several methods based on dispersion decreasing fibers (DDF) [4], soliton Raman self-scattering effect in DDF [5] or switching in a nonlinear fiber loop mirror [6]. Other techniques based on step-like and comb-like dispersion profiled fibers, using segments of conventional fibers with different dispersion, have been also proposed for the generation of picosecond and sub-picosecond pulse trains with high repetition rates [7-13]. But the above-mentioned techniques have as common feature that they require a careful control of the chromatic dispersion along the propagation distance, thus leading to relatively complicated fiber systems. An alternative approach, based on multiple four-wave mixing (FWM) in an optical fiber with anomalous constant dispersion, has been demonstrated theoretically for the generation of compressed pulse trains from the propagation of a dual-frequency pump field [14]. In particular, the optimum fiber length (propagation distance at which the pulse width is minimum) for given values of input beat signal parameters, has been accurately predicted by calculating the propagation distance where the maximum energy conversion from the pumps into the first FWM-sidebands occurs [14]. 
Relatively large compression ratios have been thus predicted theoretically, but the compressed pulses were accompanied by a strong pedestal component [14]. The objective of this letter is to present the first experimental demonstration of generation of TL pedestal-free pulse train with high repetition rate by means of the technique of multiwave mixing temporal compression in a standard non-zero dispersion-shifted fiber (NZ-DSF). In particular, in our experiments a $160-\mathrm{GHz}$ train of 1.27 ps TL pedestal-free Gaussian pulses was obtained at $1550 \mathrm{~nm}$ using a single piece of 1-km-long NZ-DSF in the anomalous-dispersion regime. The technique of frequency-resolved optical gating (FROG), adapted for high-repetition rate periodic pulse trains $[15,16]$, was used to characterize directly the intensity and phase of the $160-\mathrm{GHz}$ pulse train.

The initial beat signal is obtained by the simultaneous injection of two linearly polarized continuous waves with different frequencies and equal powers, in the NZ-DSF. The nonlinear propagation of such a beat signal was numerically analyzed using the split-step Fourier method to solve the generalized nonlinear Schrödinger (NLS) equation [17], including fiber loss and higher-order effects such as third-order dispersion (TOD), self-steepening and intrapulse Raman scattering. Figure 1(a2) shows the $160-\mathrm{GHz}$ sinusoidal input signal at 24 $\mathrm{dBm}$ average power (dashed curve) evolving into compressed pulse train with $\sim 1.3$-ps full width at half maximum (FWHM) pulses (solid curve). The optimum fiber length for achievement of maximum compression with minimum chirp and pedestal was found to be $\mathrm{L}$ $=2375 \mathrm{~m}$. At the central wavelength of the initial beat signal, i.e. $\lambda=1550.35 \mathrm{~nm}$, the fiber is assumed to have $0.21 \mathrm{~dB} / \mathrm{km}$ loss, an anomalous dispersion of $1 \mathrm{ps} / \mathrm{nm} . \mathrm{km}$, a nonlinear coefficient of $1.7 \mathrm{~W}^{-1} \cdot \mathrm{km}^{-1}$, a Raman response time of $3 \mathrm{fs}$ and a TOD of $0.07 \mathrm{ps} / \mathrm{nm}^{2} . \mathrm{km}$. These parameters are those of the NZ-DSF used in our experiments. Figure 1(a1) shows that the phase across the pulses is constant, which implies that the compressed pulses are 
transform limited. The phase difference between two consecutive pulses is $\pi$. The pulse shape corresponds essentially to a Gaussian profile with 1.34-ps FWHM. The peaks of the spectral lines of the output spectrum, shown in Fig. 1(a3), were fitted by a continuous Gaussian envelope with a FWHM bandwidth of $0.32 \mathrm{THz}$, giving a time bandwidth product of 0.43 , very close to the value of 0.441 corresponding to TL Gaussian pulses. At the average power level of $24 \mathrm{dBm}$, the multiwave mixing only slightly compresses the pulse from 3.125 ps to 1.3 ps to ensure no pedestal and no chirp. On the other hand, the compression efficiency may be enhanced by increasing the average input power as shown in Fig. 1(b2) for $30 \mathrm{dBm}$ and $\mathrm{L}$ $=977 \mathrm{~m}$. As can be clearly seen in this figure the compression efficiency is increased $(\mathrm{FWHM}=364 \mathrm{fs})$, but meanwhile the quality of the pulse train is degraded due to appearance of broad low-intensity pedestal components and a non-uniform phase across the pulses [see Fig. 1(b1)]. The corresponding spectrum in Fig. 1(b3) exhibits a slight asymmetry due to the effect of TOD. In order to describe the qualitative compression dynamics in more detail, we plotted in Figs. 1(c) and (d) the evolution with input total power of the optimum propagation distance (solid line in 1(c)), and both the output pulsewidth (solid line in 1(d)) and the proportion of pedestal energy contained in the output pulse (crosses in $1(\mathrm{~d})$ ) at the optimum fiber length. The pedestal energy is calculated by taking the relative difference between the total pulse energy and energy of an ideal Gaussian pulse having the same peak power and width. As can be clearly seen in Figs. 1(c) and (d), as the input power increases, the necessary optimum fiber length and output pulsewidth decrease, while the pulse quality is more and more degraded since the pedestal energy continually increases. Therefore we verify that pedestal-free compression is only possible at low power. On the other hand Fig. 1(c) shows that the optimum length can be accurately estimated over a wide range of input powers by calculating the length for which the maximum conversion from the two initial pumps into the first-order sidebands occurs (open circles), thus confirming the validity of the four-mode 
analytical model proposed by Trillo, Wabnitz and Kennedy [14]. Therefore, numerical solutions of the generalized NLS equation show that there exists a trade-off between pulse quality, average power, pulse-width, and fiber length.

For the experimental demonstration of this technique, we have focused our attention on the generation of TL pedestal-free pulses at $160 \mathrm{GHz}$ repetition rate. Figure 2 shows the design of the experimental setup. A dual frequency beat signal was synthesized from two cw 150-kHz-linewidth tunable-wavelength external cavity lasers (ECL1 and ECL2). The center wavelength was fixed at $\lambda=1550.35 \mathrm{~nm}$, while the zero-dispersion wavelength of the NZDSF is $\lambda_{\text {ZD }}=1536.5 \mathrm{~nm}$. Both waves were combined by a fiber fused 50:50 coupler and then amplified to the desired average power level by an Erbium-doped fiber amplifier (EDFA). Stimulated Brillouin scattering (SBS) was suppressed by externally modulating the two ECLS's with a 150-MHz sinusoidal signal applied to a $\mathrm{LiNbO}_{3}$ phase modulator. A 95:5 coupler was placed at the amplifier output to allow for real-time monitoring of the SBS signal. The two signal powers were set to the same value and the polarization states were aligned parallel. Since all devices are connected to polarization-maintaining (PM) fibers, the state of polarization of the beat signal was well defined at the NZ-DSF input. The beat signal power injected into the 1-km-long NZ-DSF was set to $27.2 \mathrm{dBm}$, which leads to optimum compression with minimum pedestal. The output field after nonlinear reshaping in the NZDSF, was characterized with an optical spectrum analyzer (OSA), a background-free secondharmonic generation (SHG) autocorrelator and a FROG setup based on the spectral analysis of the SHG autocorrelation signal with the OSA. The FROG trace was conveniently built up by adjusting the autocorrelator to a particular delay, and then scanning the OSA wavelength. The measured temporally-modulated spectra were then interpolated onto a $128 \times 128$ grid, 
and used as input to the vector-based principal components generalized projections (PCGP) algorithm for the retrieval of the periodic pulse-train characteristics $[15,16]$.

Figures 3(a) and (b) show, respectively, the measured and retrieved SHG-FROG traces of the field after propagation through the 1-km NZ-DSF. The FROG trace exhibits a complex structure because of the generation of multiple FWM sidebands associated with the reshaping of the injected beat signal. The retrieval error, defined as the $r m s$ between the original and retrieved FROG traces, was found to be $\mathrm{G}=0.0035$, which is a small error for this complex form of periodic FROG trace in the presence of noise. The corresponding retrieved intensity and phase are shown in Fig. 3(c). Remarkably, the intensity profile shows reshaped pulse train with clearly no pedestal. The phase variation over the compressed pulses forming the train is also negligibly small, indicating that the pulses are essentially transform limited. The phase difference between two consecutive pulses is $\pi$, in good agreement with theoretical predictions of Fig. 1. The solid line in Fig. 3(d) is the least squares fit of a Gaussian function to the retrieved pulse shape. As can be clearly seen in this figure, the intensity profile corresponds very well to a Gaussian function with 1.27-ps FWHM and 2.4-W peak power. The extinction ratio between peak power and interpulse background is better than $20 \mathrm{~dB}$. The pulse duration achieved corresponds to a duty cycle of 5:1. As shown on a logarithm scale in Figs. 3(e) and (f) respectively, the autocorrelation and the spectrum that were derived from the retrieved pulse train (circles) are in excellent agreement with the independent direct experimental measurements (solid lines), thus confirming the reliability of the experimental setup and the PCGP retrieval algorithm. Our experimental results are in qualitatively good agreement with the numerical solutions of the generalized NLS equation as shown in Fig. 1. However a more direct comparison between theory and experiments would require perfect 
knowledge of the fluctuation of the physical parameters along fiber length, such as dispersion, nonlinearity and birefringence.

In conclusion, we have presented the experimental demonstration of highly efficient multiwave mixing compression of a sinusoidal beat signal into a high-quality train of Gaussian 1.27-ps TL pedestal-free pulses at 160-GHz repetition rate and $1550 \mathrm{~nm}$. The FROG technique has been proven to be a powerful tool to accurately characterize the intensity and phase of the $160-\mathrm{GHz}$ pulse train. For an industrial application of this technique, the timing jitter that originates from fluctuations of frequency difference between the two light sources can be reduced by stabilizing the two external cavity lasers through a single reference cavity, such as a Fabry-Perot resonator with a very high finesse [18]. Finally, we believe that the very simple experimental technique described in this letter exhibits great potential as a high-quality source of TL ultra-short pulses, which can be helpful for optimizing ultra-high bit-rate transmission lines.

Guy Millot's e-mail address is Guy.Millot@u-bourgogne.fr. 


\section{References}

1. T. Yamamoto, E. Yoshida, K.R. Tamura, K. Yonenaga, and N. Nakazawa, IEEE Photon. Technol. Lett. 12, 353 (2000).

2. A. Hasegawa, Opt. Lett. 9, 288 (1984).

3. K. Tai, A. Tomita, J.L. Jewell and A. Hasegawa, Appl. Phys. Lett. 49, 236 (1986).

4. P.V. Mamyshev, S.V. Chernikov, and E.M. Dianov, IEEE J. Quantum Electron. 27, $2347(1991)$.

5. S.V. Chernikov, D.J. Richardson, R.I. Laming, E.M. Dianov, and D.N. Payne, Appl. Phys. Lett. 63, 293 (1993).

6. S.V. Chernikov and J.R. Taylor, Electron. Lett. 29, 658 (1993).

7. S.V. Chernikov, J.R. Taylor, and R. Kashyap, Electron. Lett. 29, 1788 (1993).

8. S.V. Chernikov, J.R. Taylor, and R. Kashyap, Electron. Lett. 30, 433 (1994).

9. S.V. Chernikov, J.R. Taylor, and R. Kashyap, Opt. Lett. 19, 539 (1994).

10. S.V. Chernikov, R. Kashyap, M.J. Guy, D.G. Moodie, and J.R. Taylor, Phil. Trans. R. Soc. Lond. A 354, 719 (1996).

11. E. A. Swanson and S.R. Chinn, IEEE Photon. Technol. Lett. 7, 114 (1995).

12. A. Maruta, Y. Yamamoto, S. Okamoto, A. Suzuki, T. Morita, A. Agata, and A. Hasegawa, Electron. Lett. 36, 1947 (2000).

13. M. Tadakuma, O. Aso, and S. Namiki, in Optical Fiber Communication Conference, 2000 OSA Technical Digest Postconference Edition vol. 3 (Optical Society of America, Washington, D.C., 2000), pp. 178-180.

14. S. Trillo, S. Wabnitz, and T.A.B. Kennedy, Phys. Rev. A 50, 1732 (1994).

15. F. Gutty, S. Pitois, P. Grelu, G. Millot, M.D. Thomson, and J.M. Dudley, Opt. Lett. 24, 1389 (1999). 
16. J.M. Dudley, F. Gutty, S. Pitois, and G. Millot, IEEE J. Quantum Electron. 37, 587 (2001).

17. G.P. Agrawal, Nonlinear Fiber Optics, 3rd ed. New York: Academic (2001).

18. A. Schoof, J. Grünert, S. Ritter, and A. Hemmerich, Opt. Lett. 26, 1562 (2001). 


\section{Figure captions}

Fig. 1. Results of numerical simulation of a beat signal transformation into a train of short pulses for an input average total power of $24 \mathrm{dBm}(0.25 \mathrm{~W})$. (a1) Phase variation. (a2) Intensity of the compressed pulse train (solid curve) and the input beat signal (dashed curve). (a3) Spectrum of the compressed pulse train. Here the zero frequency corresponds to the central wavelength of the initial beat signal. Figs. (b1), (b2) and (b3) show the results for the pulse train compression with an input average power of $30 \mathrm{dBm}(1 \mathrm{~W})$. (c) Optimum length versus the input total power. (d) Output pulsewidth (solid line) and proportion of pedestal energy (crosses) at the optimum fiber length.

Fig. 2. Schematic of the experimental setup. ECL: external cavity laser. PM: polarization maintaining. EDFA: Erbium-doped fiber amplifier. ATT: variable attenuator. PC: polarization controller. OSA: optical spectrum analyzer.

Fig. 3. (a) Measured and (b) retrieved SHG-FROG traces of the generated pulse train at 160 GHz. (c) The solid line shows the retrieved intensity (left-hand axis), whilst the dashed line shows the retrieved phase (right-hand axis). (d) Least squares fit of the retrieved pulse shape (open circles) by a Gaussian function (solid line). (e) Measured background-free intensity autocorrelation and (f) spectrum of the 160-GHz pulse train at fiber output (solid curves). The open circles show the autocorrelation and spectrum calculated from the retrieved intensity and phase. 

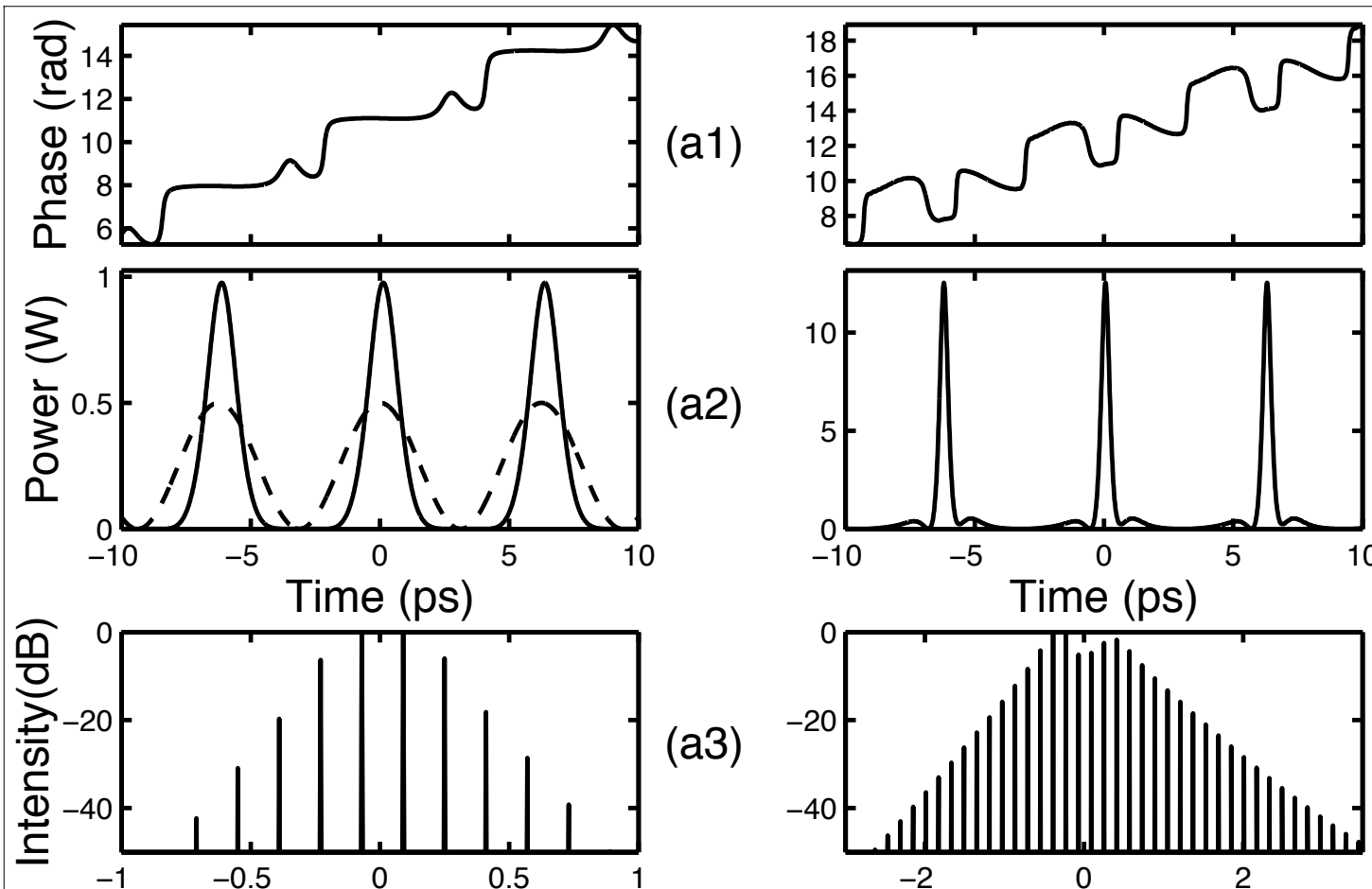

Frequency detuning $(\mathrm{THz})$

(a3)

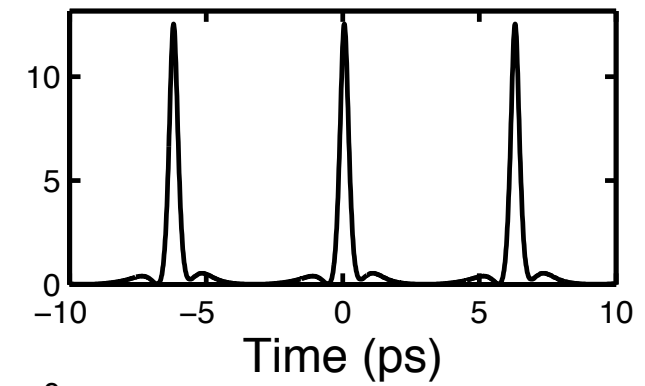

(b2)

(a2)
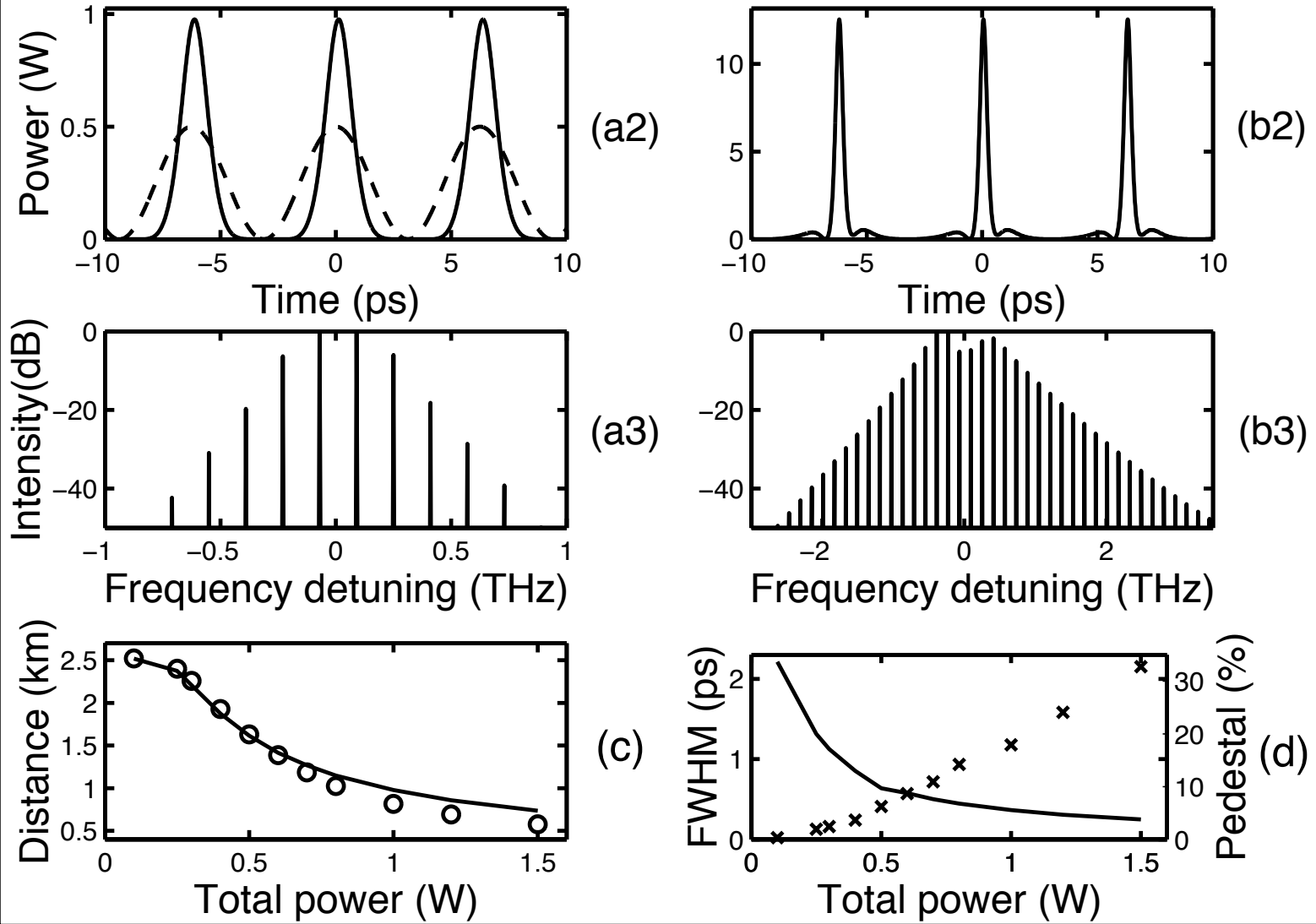
Fig. 2

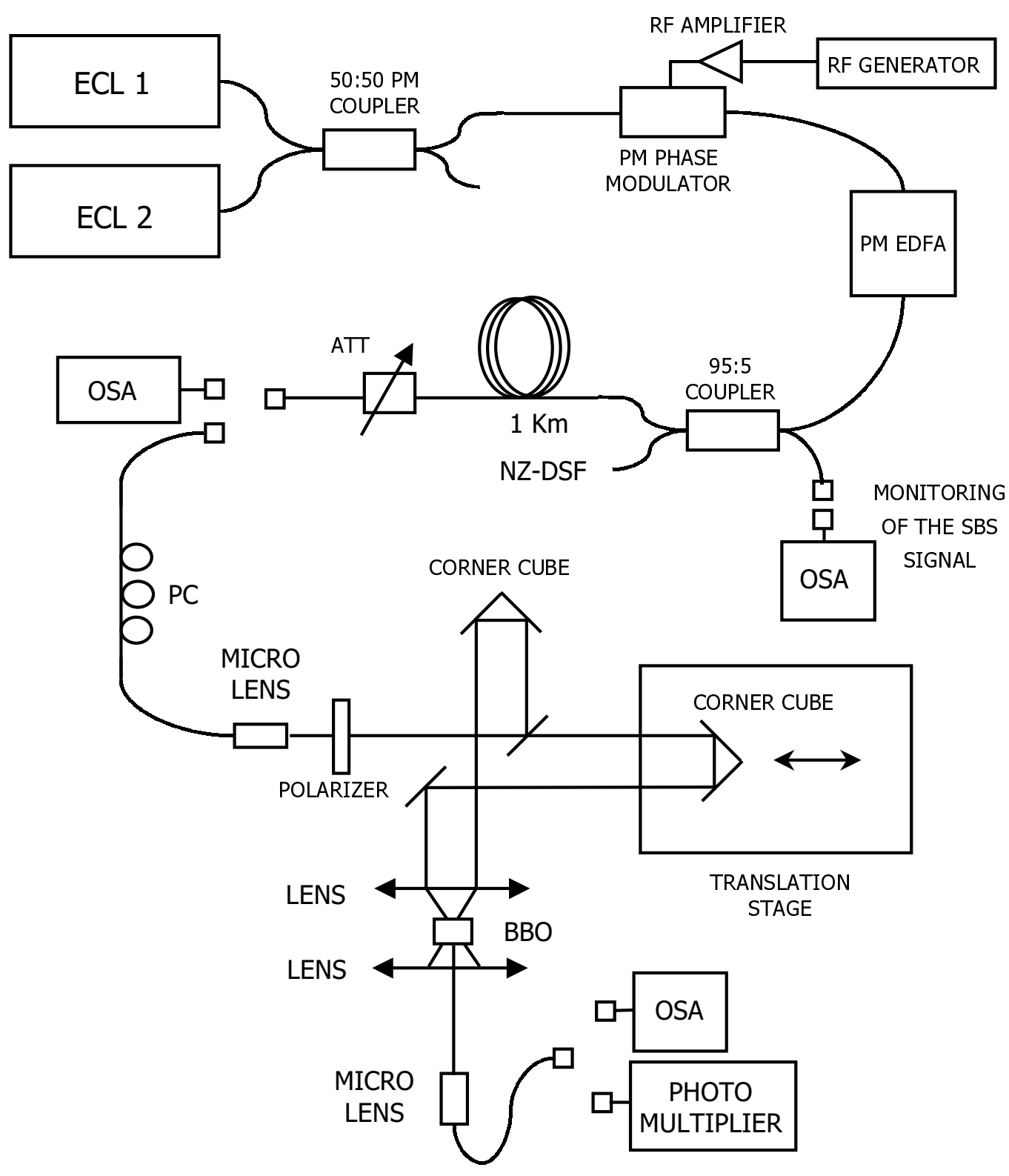



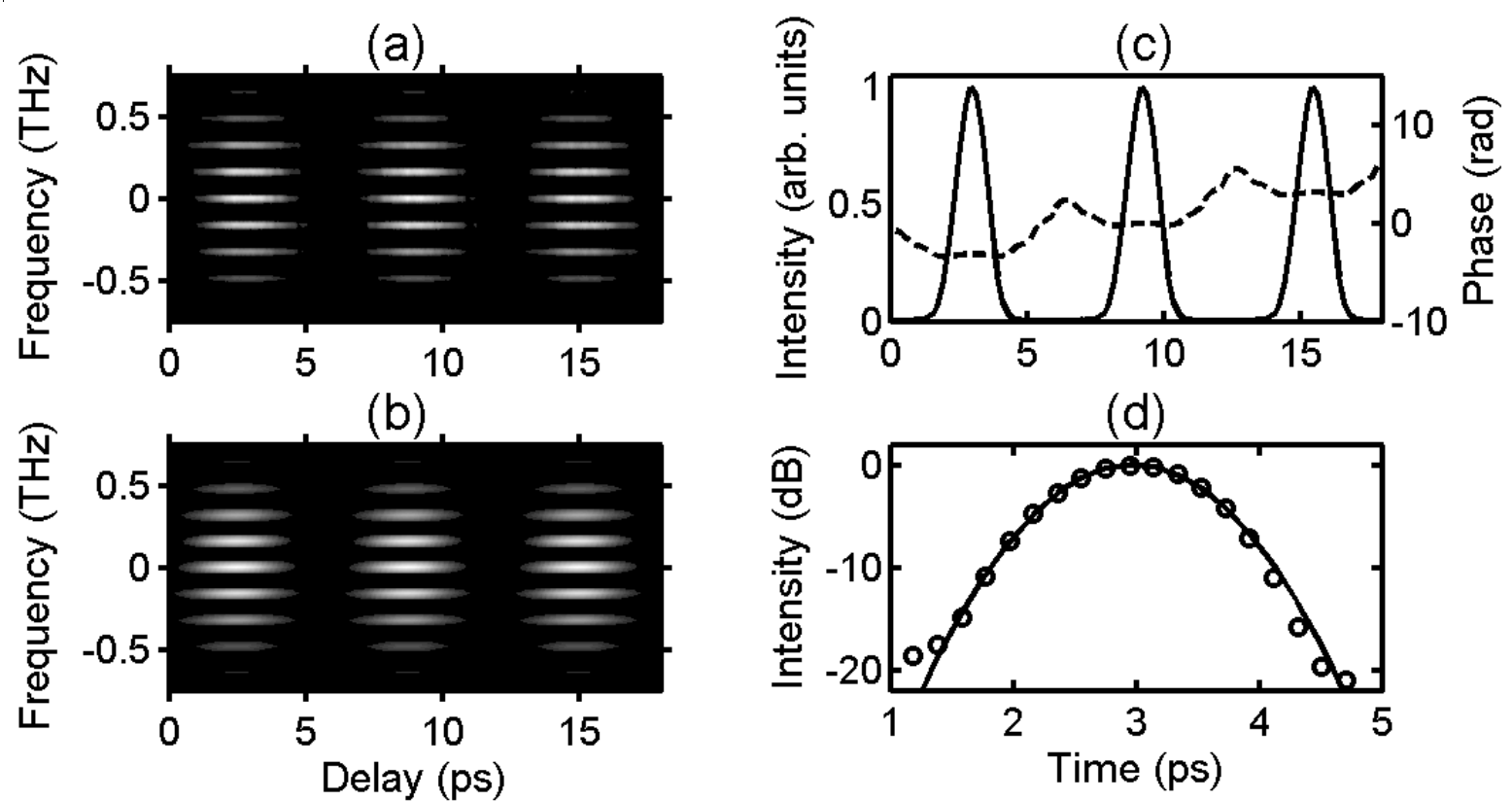

(d)

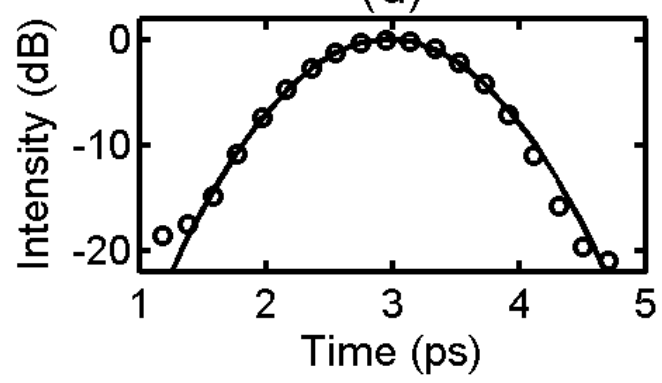

(e)
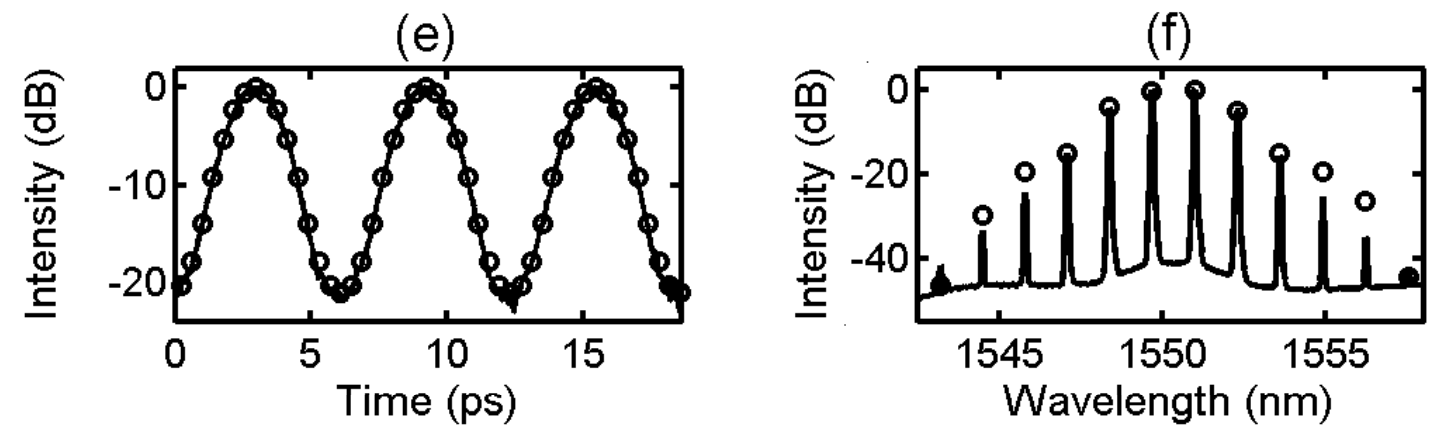